\title{
Bioinformation
}

\section{Breaking the HxNy outbreak}

\author{
Jayapal Manikandan ${ }^{1}$, Amudala Hemanthakumar Karthik ${ }^{2}$, Peter Natesan Pushparaj ${ }^{2 *}$ \\ ${ }^{1}$ Department of Physiology, Yong Loo Lin School of Medicine, National University of Singapore, Singapore-119260, ${ }^{2}$ Division of Immunology, \\ Infection and Inflammation, Glasgow Biomedical Research Centre, University of Glasgow, Glasgow, G12 8TA, United Kingdom. ${ }^{2}$ Division of \\ Immunology, Infection and Inflammation, Glasgow Biomedical Research Centre, 120 University Place, University of Glasgow, Glasgow, G12 \\ 8TA, Scotland, United Kingdom. Peter Natesan Pushparaj - Email: pnp1j@clinmed.gla.ac.uk, *Corresponding Author
}

Received October 10 2009, Accepted November 14 2009, Published February 28, 2010

\begin{abstract}
The latest emergence of influenza A (H1N1) virus outbreak demonstrated how swiftly a new strain of flu can evolve and spread around the globe. The A/H1N1 flu has been spreading at unprecedented speed, and further spread within the countries being affected and to other adjacent or far way countries is considered inevitable due to the rapid emigration of infected individuals across the world. In this bioinformation, we discuss the mechanism of evolution of a new HxNy strain and the essential criteria for potentially breaking the outbreak of these extremely harmful and rapidly evolving viral strains in the near future by taking the recent H1N1 pandemic as a classical paradigm.
\end{abstract}

Key Words: Influenza A, HxNy, H1N1, Pandemic, Outbreak

\section{Background:}

The emergence of influenza A (H1N1) 9 decades ago led to a devastating global pandemic. H1N1 is thought to have emerged almost simultaneously from birds into humans and swine [1]. As of July 23, 2009, the H1N1 virus was reported in more than 130 countries, with more than 800 deaths. Several countries are experiencing sustainable community transmission. The sudden outbreak of this novel flu virus has tested the world's public health preparedness. H1N1 provided a real-world test that showed the strengths and vulnerabilities in the abilities of the countries in world to respond to a major infectious disease outbreak [2]. The scale of the problem is uncertain when a disease first appears but increased rapidly. Pandemic preparations will be severely challenged when the pandemic eventually arrives to countries, especially if there is high morbidity and mortality. Healthcare facilities can be quickly overwhelmed and the economy can go into deep recession. There can be widespread public anxiety and fear. It is thus vital to ensure that preparedness plans are regularly reviewed and updated to incorporate the latest research findings and technology where feasible. Equally important are exercises to test and fine-tune procedures and plans. The consequences of $\mathrm{H} 1 \mathrm{~N} 1$ have been less severe than originally anticipated, but there are some important lessons to be learned. Should the worst occur, most countries would be caught without the necessary policies and plans in place to deal with a deadly outbreak. Early pandemic preparation is required, but decisions about action must be made when the threat is only modest. Major intervention may cause such as rare adverse events from large-scale vaccination or economic and social costs from school dismissals and the uncertain probability of much greater harm from a widespread outbreak. This combination of urgency, uncertainty, and the costs of interventions makes the effort to control infectious diseases especially difficult [3]. This bioinformation examines underlying concerns and lessons learned from the response about overall preparedness for potential pandemic flu outbreak in the event that H1N1 returns in the future, either in its current form or as a more virulent strain.

\section{HxNy Pandemic and Social preparedness:}

If a severe pandemic occurs, it is likely to be a prolonged and widespread outbreak that could require major changes in many sectors of society, such as schools, work, transportation, business, health care, and government. The public can greatly reduce their risk during a pandemic by being informed and prepared before the emergency.

\section{Social isolation:}

Nonmedical interventions primarily social distancing could be useful in staving off transmission [4]. Limiting social contact will slow the spread of influenza in the community and will reduce the height of the peak of the epidemic, although overall attack rates may remain the same. This will help healthcare services to cope and minimize the likelihood that healthcare services would be overwhelmed. Social distancing measures will take the form of closure of all educational institutions and most public places e.g. shopping malls, cinemas and swimming pools. Public gatherings at mass events such as concerts and sporting events will also be cancelled. Schools and corporate finds more ways to be flexible with their work-from-home options [5].

\section{Delaying the spread of $\mathrm{HxNy}$ :}

At the earliest sign that an influenza pandemic has begun (Figure 1), border controls would be stepped up in an attempt to delay the spread of the pandemic virus. It has been reported that such measures may have limited usefulness but measures such as temperature and health screening were used during the SARS epidemic were so effective and it is good to be employed again during an influenza pandemic [6]. Depending on the severity of the pandemic, other border control measures that could be implemented include the imposition of immigration visa requirements and quarantine of incoming travelers, including residents. These would minimize non-essential travels [7].

\section{Planning and management:}

Improving coordination among governments and the private sector preparedness and planning activities are critical in future preparation as the nature of flu threats change over time. Communication between the public health system and health providers must be well coordinated. Building trust, technologies, and policies internationally to encourage science-based, consistent decision making across globally during an outbreak is very important.

\section{Super-flexible response strategy:}

As the epidemic unfolded, new knowledge required government officials to reassess guidance offered to the public and the medical community. Providing clear, straightforward information to the public is essential for allaying fears and building trust gives people the facts they need to be prepared, not scared.

\section{Maintaining indispensable services:}

Improving the ability for health providers to manage a massive influx of patients is essential. Even with a mild outbreak, the health care delivery system was overwhelmed. It revealed significant problems and lack of preparedness. Investments in antiviral stockpiles and enhanced vaccine manufacturing capacity also proved to be prudent. Countries need to make sure that they have enough stockpiles of food essentials such as rice and infant milk powder. 


\section{Bioinformation}

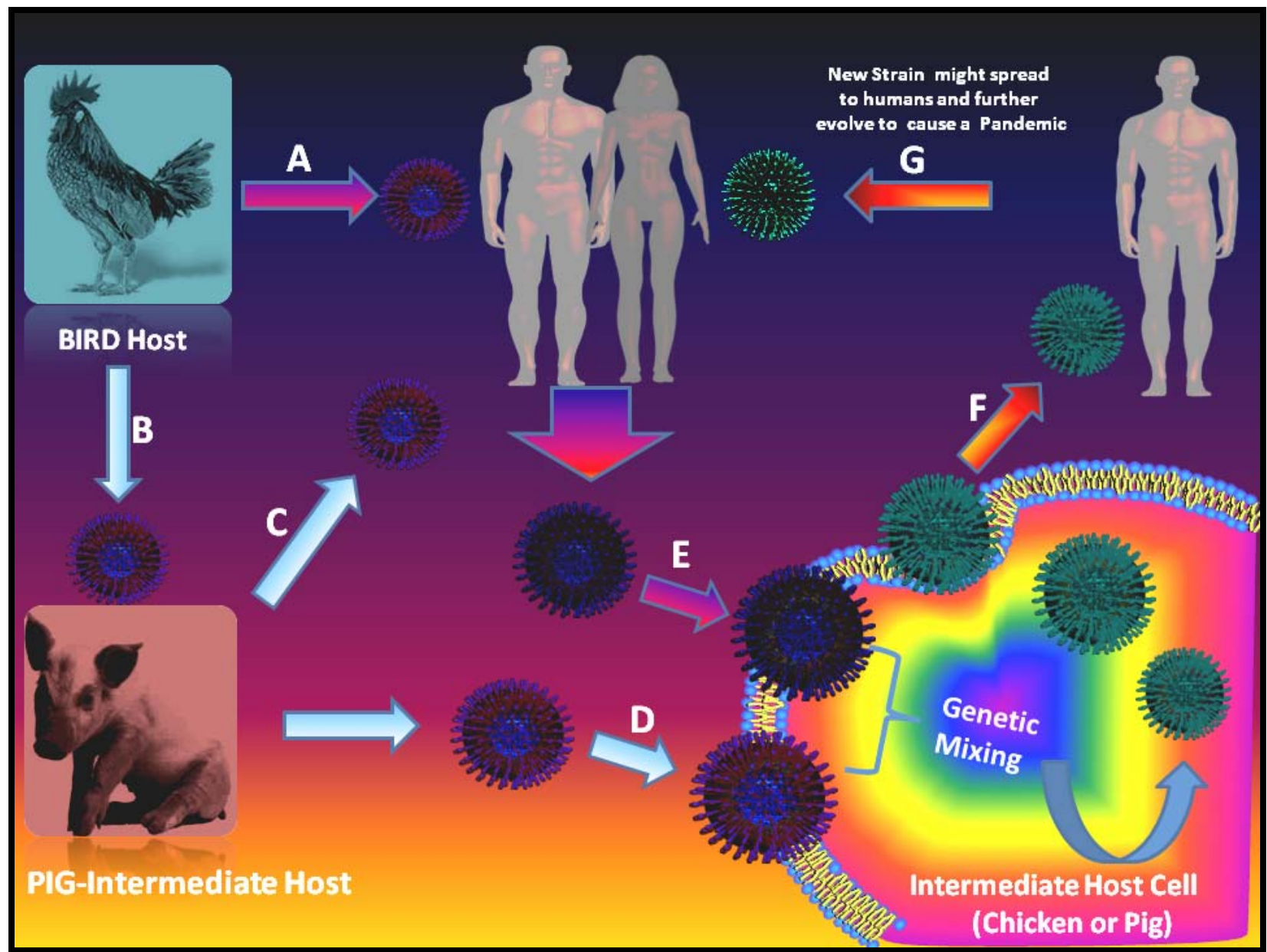

Figure 1: Mechanism of HxNy Evolution. Influenza virus A subtypes, belongs to the family of orthomyxoviridae, are classified as H1N1, H1N2, H3N2 or H1N5 etc., based on 16 Hemaglutinin (H) serotypes or subtypes (imputed for $x$ variable) and from 9 subtypes of neuraminidase (N) (imputed for $y$ variable) on the viral surface. However, the specific influenza strain isolates are identified by a standard nomenclature specifying virus type, geographical location where first isolated, sequential number of isolation, year of isolation, and also the HA and NA subtypes. (A) Without any genetic modification influenza A virus can be passed on to humans. (B) Birds or aquatic birds can also spread influenza A subtypes directly to pigs. (C) Pigs, which are one of the intermediate hosts as birds can pass on the virus to humans. (D)However, inside an intermediate host like pig, a bird influenza virus can be genetically mixed with a human strain of influenza virus subtype since humans can transmit the virus directly to pigs (E) causing the evolution of a new virulent strain of influenza which might infect the humans to cause an epidemic (F) and further spreading of this new strain in different human populations across the globe will eventually lead to a full fledged flu pandemic (G).

\section{Trade limitations:}

There is no scientific evidence that eating pork causes influenza. Yet 20 countries have banned meat from Mexico, Canada, and the United States, including 2 of the world's largest pork importers, China and Russia. However, more scientific evidence required whether such bans are necessary to implement during pandemic outbreak.

\section{Pandemic drills:}

Government agencies have to carry out exercises to test their pandemic preparedness plans. The exercise will be useful in enabling healthcare providers to fine-tune their operational plans for an influenza pandemic. As seen for the past few weeks, social media such as twitter, blogs and social networks, can play a vital role in the dissemination of information. A public exhibition on infectious diseases, including flu pandemics will raise public consciousness about the impact of major epidemics and the need for tough measures to be taken.

\section{Additional procedures:}

Other measures include public advice on good personal hygiene such as good cough etiquette and frequent washing of hands. The public could also be advised to stay home as much as possible and wear surgical masks whenever they are in public places including when travelling on public transportation. Members of households with influenza cases will be asked to practice voluntary home quarantine to prevent possible transmission. Useful lessons were learnt during SARS such as transparency and the provision of regular updated information as well as gene expression profiling data from the SARS patients which are essential to design a novel treatment regimen for containing the outbreak $[\mathbf{8}, \mathbf{9}]$. Another lesson is that just because SARS started in Asia doesn't mean the next bug will be born there. A potential pandemic can originate and take root anywhere [10].

\section{Pandemic flu and medical preparedness}

The emergence of yet another serious global health threat from an animal source highlights the critical need for deeper understanding of zoonotic viruses, including in vivo studies of pathogenesis in animals, 


\section{Bioinformation}

field epidemiologic studies, and surveillance in animal populations, along with the development of computational models. The shift in mortality toward younger age groups was the most striking characteristic of the 20th-century pandemics [11, 12]. Data from past pandemics can provide useful insights for current and future planning [13]. Though the rapidity of transmission of influenza virus during pandemics necessitates immediate action, it can be hoped that close collaborations and lessons drawn from previous pandemics will contribute to reducing national and global mortality. The documented relevant signature features can help health authorities prioritize national strategies and aid international collaborators in addressing the initial and successive waves of illnesses and deaths.

\section{National stockpile:}

Maintaining enough stockpiles to make sure enough antiviral medications, vaccinations, and equipment are available to protect people. During the SARS epidemic in 2003, personal protective equipment (PPE) such as masks, gloves and waterproof gowns were in short supply.

Prophylaxis for health care professional:

It is important to make sure that there is sufficient medication provided in advance to front-line health care professionals. For example, doctors and their staffs might need numerous 10-day courses of antiviral for them to continue treating patients for the duration of a pandemic without getting sick. Workers in essential services such as healthcare, energy supply, water supply, and law enforcement will be provided prophylaxis with oseltamivir to minimize their likelihood of being infected with influenza.

\section{HxNy research initiatives:}

Providing enough funding for the future research on H1N1 is very important Identification of pre-1918 human influenza RNA samples would help us understand the timing of emergence of the 1918 virus. Understanding influenza pandemics in general requires understanding the 1918 pandemic in all its historical, epidemiologic, and biologic aspects [14]. It is also important to enhance the biomedical research and development abilities to rapidly develop and produce a vaccine.

\section{Case definition and testing methods:}

Countries have reported that the clinical case definition was not an accurate model for the swine flu epidemic; and drug and mask distribution has been extremely slow, especially to general practitioners, who are crucial to effective disease management and containment [15]. Initial laboratory testing to confirm a case of swine flu was only allowed if a sick patient has been recently returned from affected countries. This is the opposite of what has occurred. To understand the full extent of disease spread, widespread testing without assumptions needs to occur so that the accuracy of the proposed case definition can be tested.

\section{Drug resistance and alternative therapy:}

It is shown that the main seasonal influenza strain in the United States in 2008 was resistant to oseltamivir in 0.4 percent to 4 percent of adults and children treated [16]. The real nightmare scenario will be if the current swine flu strain also develops resistance in the face of widespread use of oseltamivir, leaving us with few treatment options.

\section{Conclusion:}

The documented relevant signature features can help health authorities prioritize national strategies and aid international collaborators in addressing the initial and successive waves of illnesses and deaths. The recent H1N1 Influenza outbreak raised concerns about our ability to handle the current and future pandemic threats. Our systems for surveillance, reporting, infection control, and antiviral drug distribution showed strain and could potentially be overwhelmed. In the brief time we have to prepare for our next wave, we must step back and evaluate where improvements need to be made. Preparations must continue to be improved and fine-tuned to ensure that the impact of the future influenza pandemic is minimized as much as possible. It is important to plan for the constant evolution of H1N1 virus. H1N1 may transform into a deadly global threat at some point, debilitating normal life across the world. Countries must heed the lessons of this scare and recognize that preparedness in the present is essential. By implementing comprehensive policies and investigating the strengths and weaknesses before any real crisis hits, we can ensure a relatively smooth transition into a state of emergency, should they be faced with the worst. Though the rapidity of transmission of influenza virus during pandemics necessitates immediate action, it can be hoped that close collaborations and lessons drawn from the recent H1N1 pandemic will contribute to reducing national and global mortality.

\section{Acknowledgement}

The authors wish to thank "Beacon Biosoft Pvt.Limited" (www.beaconbiosoft.com) for helping with the wonderful graphics on the Mechanism of HxNy Evolution in this article.

\section{References:}

[1] SM Zimmer, DS Burke. $N$ Eng J Med. (2009) 361:279. [PMID: 19564632].

[2] http://www.who.int/mediacentre/news/statements

[3] M Lipsitch et al., $N$ Eng J Med. (2009) 361:112. [PMID: 19474417].

[4] C Viboud et al, Vaccine (2006) 24:6701. [PMID: 16806596]

[5] http://www.ed.gov/admins/lead/safety/emergencyplan/pandemic /guidance/flu-faqs.pdf

[6] NM Ferguson et al., Nature (2006) 442: 448-52 [PMID: 16642006].

[7] http://news.bbc .co.uk/2/hi/americas/8022516.stm

[8] PN Pushparaj et al., Nature Precedings (2007) 1145.1.

[9] R Reghunathan et al., BMC Immunology. (2005) 6:2. [PMID: 15655079]

[10] DJ Cleri et al., Infect Dis Clin North Am (2010). 24:175-202. [PMID: 20171552]

[11] L Simonsen L et al., Washington, DC: National Academies Press, (2005): 89.

[12] V Andreasen et al., J Infect Dis (2008) 197:270-8. [PMID: 18194088]

[13] MA Miller et al., $N$ Eng J Med. (2009) 360:2595-8. [PMID: 19423872]

[14] JK Taubenberger, DM Morens. Emerg Infect Dis.(2006)12:1522.[PMID: 16494711]

[15] A Swaminathan et al., Emerg Infect Dis (2007) 13: 1541-1547.

[16] http://www.recombinomics.com/News/07030903/H274Y_Lesso ns.html.

Edited by P. Kangueane Citation: Manikandan et al., Bioinformation, 4(8) 338-340 (2010) License statement: This is an open-access article, which permits unrestricted use, distribution, and reproduction in any medium, for noncommercial purposes, provided the original author and source are credited. 